\title{
Crescimento inicial, produção e qualidade de frutos de macieiras submetidas a irrigação e fertirrigação
}

\author{
Initial growth, production, and quality of fruits of apple trees as affected by irrigation and fertirrigation
}

\section{Maêve Silveira Castelo Branco ${ }^{1 *}$, Gilberto Nava² e Paulo Roberto Ernani ${ }^{1}$}

Recebido em 05/05/2015 / Aceito em 01/02/2016.

\section{RESUMO}

O presente trabalho objetivou avaliar o desenvolvimento inicial da macieira, os aspectos nutricionais, qualidade dos frutos em função da forma de aplicação dos fertilizantes e da adição ou não de água via irrigação. O experimento foi conduzido em São Joaquim durante as estações de crescimento 2012/2013 e 2013/2014 em pomar implantado no ano de 2011 com a cultivar 'Kinkas', sobre porta-enxerto Marubakaido com interenxerto M-9, na densidade de 1.666 plantas ha ${ }^{-1}$. Utilizouse o delineamento experimental de blocos ao acaso, com oito repetições dos seguintes tratamentos: T1 adubação convencional; T2 - irrigação + adubação convencional; T3 - irrigação + fertirrigação; e T4 fertirrigação. Através da média das leituras diárias dos tensiômetros, e a curva de retenção de água no solo foi determinada a necessidade de irrigação e o tempo de aplicação. Em 2012, foram aplicados $27 \mathrm{~kg} \mathrm{ha}^{-1}$ de $\mathrm{N}$, e na safra seguinte $100 \mathrm{~kg} \mathrm{ha}^{-1} \mathrm{de} \mathrm{Ne} 150 \mathrm{~kg} \mathrm{ha}^{-1} \mathrm{de}$ $\mathrm{K}_{2} \mathrm{O}$. A adubação foi parcelada em três aplicações para T1 e T2, e seis aplicações quinzenais para T3 e T4, no período de novembro a janeiro. Durante o período experimental foram observados vários períodos de déficit hídrico. O uso de irrigação e/ou fertirrigação não influenciou o desenvolvimento inicial de macieiras. Não houve acréscimo nos teores foliares e da polpa dos frutos para os nutrientes avaliados em resposta ao uso de irrigação para a macieira. A avaliação dos frutos demonstrou que a adubação convencional apresentou maior firmeza de polpa. Os frutos do tratamento irrigação + fertirrigação apresentaram o maior peso médio, porém a produtividade não diferiu entre os tratamentos. A fertirrigação demonstrou ser a melhor forma de aplicação de fertilizantes, mas mesmo assim é necessário aprimorar as doses e períodos de aplicação para obter máxima eficiência produtiva.

PALAVRAS-CHAVE: Déficit hídrico, composição mineral, Malus domestica Borkh.

\section{ABSTRACT}

This study aimed to evaluate nutrient content on leaves and fruit flesh, yield and quality of fruits, as well as apple tree growth as affected by the fertilizer physical form (conventional or fertirrigation) and water addition or not via irrigation. The experiment was carried out in São Joaquim, Southern of Brazil, during the 2012/2013 and 2013/2014 growing season. The orchard was planted in 2011 with 'Kinkas' cultivar grafted over a Marubakaido rootstock, 1.666 trees $\mathrm{ha}^{-1}$. The eight replications of each treatment were allocated in the completely randomized block design. Treatments consisted of conventional solid soil fertilization (T1), irrigation + conventional solid soil fertilization (T2), irrigation + fertirrigation (T3), and fertirrigation (T4). From the daily averages of tensiometer readings in addition to the water soil retention the irrigation needs and the application time curve were determined. In 2012, $27 \mathrm{~kg} \mathrm{ha}^{-1}$ of $\mathrm{N}$ was applied; in the following season, it $100 \mathrm{~kg} \mathrm{ha}^{-1}$ of $\mathrm{N}$ and $150 \mathrm{~kg} \mathrm{ha}^{-1}$ of $\mathrm{K}_{2} \mathrm{O}$ was applied. Fertilizers were split three times on treatments $\mathrm{T} 1$ and $\mathrm{T} 2$, and six times on treatments $\mathrm{T} 3$ and $\mathrm{T} 4$, from November through January. During the experimental period it many drought periods were observed. Irrigation and fertirrigation had no effect on initial apple tree growth. Irrigation did not affect the nutrient content in leaves or fruit flesh. Fruits from the T1 treatment had higher firmness than fruits from the other treatments. Fruits from T3 (irrigation + fertirrigation) presented the highest weight. Fruit yield, however, did not differ

${ }^{1}$ Universidade do Estado de Santa Catarina, Lages, SC, Brasil.

${ }^{2}$ Empresa Brasileira de Pesquisa Agropecuária, Pelotas, RS, Brasil.

*Autor para correspondência <maevecastelo@hotmail.com>. 
among treatments. Fertirrigation was the best form of nutrient application to the plants, but it is necessary to determine rates and times of application to obtain the maximum productive efficiency.

KEYWORDS: Water deficit, mineral composition, Malus domestica Borkh.

\section{INTRODUÇÃO}

Embora as principais regiões produtoras de maçãs do sul do Brasil apresentem elevados índices pluviométricos, acima de $1200 \mathrm{~mm}$ anuais, durante o ciclo da cultura é comum a ocorrência de déficit hídrico o que pode prejudicar a qualidade dos frutos e a produtividade (CONCEIÇÃO et al. 2009). Além disso, favorece o desenvolvimento de distúrbios fisiológicos principalmente quando o déficit ocorre no início do desenvolvimento dos frutos, fase em que há maior absorção de cálcio, e a diferenciação floral para o ciclo seguinte (PETRI 2006). Sendo assim, a utilização de sistemas de irrigação tem a função de complementar o fornecimento de água e nutrientes durante períodos de déficit hídrico para que a produção não seja prejudicada.

De maneira geral, o sistema de irrigação por gotejamento é o mais recomendado para pomares de maçã modernos, pois promove maior economia de água, produtividade e qualidade dos frutos (FALLAHI et al. 2010, FALLAHI 2012). O monitoramento da tensão da água no solo, visando uma correta aplicação de irrigação, é eficiente para manter os índices de umidade do solo adequados para a cultura (CONCEIÇÃO et al. 2011).

O momento ideal de aplicação dos fertilizantes para suprir as necessidades da planta e proporcionar o crescimento e desenvolvimento dos frutos, sem causar desequilíbrio nutricional, depende da mobilidade do nutriente no solo. A fertirrigação, é um método eficiente de fornecimento de nutrientes para árvores frutíferas (NEILSEN et al. 1998), pois disponibiliza nutrientes dissolvidos na água de irrigação, que podem ser prontamente absorvidos pelas plantas. NEILSEN \& NEILSEN (2008) consideram que a fertirrigação permite maior flexibilidade no período de aplicação, e na precisão do fornecimento de nutrientes, independente das condições climáticas (KIPP 1992). A disponibilidade de nutrientes pouco móveis no solo em direção às raízes, como $\mathrm{P}$ e $\mathrm{K}$, é facilitada quando estes nutrientes são aplicados em solução, via fertirrigação (NEILSEN et al. 1999, NEILSEN \& NEILSEN 2008), o que também pode favorecer a movimentação no perfil do solo (SOUZA et al. 2006). Segundo KIPP (1992) a fertirrigação pode ser utilizada como medida para reduzir o período improdutivo nos pomares jovens.

O manejo da irrigação em regiões de clima temperado, tropical e subtropical que apresentam precipitação pluviométrica em volumes adequados, porém mal distribuídos, ainda não está bem estabelecido (AL-YAHYAI 2012).

O objetivo deste trabalho foi avaliar o efeito da irrigação, bem como comparar o efeito da adubação convencional (em superfície) em relação ao uso da fertirrigação quanto ao crescimento inicial de macieiras, teor de nutrientes nas folhas e na polpa dos frutos, incremento da produtividade e qualidade dos frutos 'Kinkas' cultivadas em São Joaquim- SC.

\section{MATERIAL E MÉTODOS}

O experimento foi conduzido na Estação Experimental da EPAGRI de São Joaquim - SC (2816'30'S, 4955'53'W - altitude de $1400 \mathrm{~m}$ ), durante as estações de crescimento 2012-2013 e 20132014.O pomar foi plantado no ano de 2011 em um Cambissolo Húmico. Utilizou-se a cultivar Kinkas, sobre porta-enxerto Marubakaido com interenxerto M-9. O sistema de condução utilizado foi o de líder central, na densidade de 1.666 plantas por hectare.

$\mathrm{Na}$ implantação do experimento foi realizada a correção da acidez para elevar o $\mathrm{pH}_{\mathrm{H} 2 \mathrm{O}}$ do solo para 6,5 e a adubação corretiva da fertilidade do solo com $\mathrm{K}_{2} \mathrm{O}$ e $\mathrm{P}_{2} \mathrm{O}_{5}$, de acordo com a recomendação da CQFS-RS/SC (2004).

O delineamento experimental foi o de blocos ao acaso, com oito repetições dos seguintes tratamentos: T1 - adubação convencional; T2 - irrigação + adubação convencional; T3 - irrigação + fertirrigação; e T4 - fertirrigação. As parcelas foram compostas por seis plantas, sendo consideradas as quatro centrais como úteis.

Em outubro de 2012 foi instalado o sistema de irrigação localizada por gotejamento, sendo utilizadas duas mangueiras em cada fila, uma para irrigação e outra para fertirrigação. Os gotejadores foram espaçados em 0,60 m, com vazão de 1,6 litros por hora. Tensiômetros de punção foram instalados entre as plantas centrais de cada parcela (em seis dos oito blocos do experimento), nas profundidades de $15 \mathrm{e}$ 
$30 \mathrm{~cm}$. Utilizando a média das leituras diárias dos tensiômetros e a curva de retenção de água no solo, foi determinada a necessidade de irrigação e o tempo de aplicação para manter a tensão do solo em $-10 \mathrm{kPa}$ (CC).

Foram aplicadas as mesmas quantidades de nutrientes ( $\mathrm{N}$ e $\mathrm{K}$ ) em todos os tratamentos, diferindo apenas quanto à forma física de aplicação dos fertilizantes (convencional e via fertirrigação), e à frequência das aplicações. Na safra 2012-2013 realizou-se apenas adubação nitrogenada de cobertura utilizando-se ureia em todos os tratamentos. Aplicouse $27 \mathrm{~kg} \mathrm{ha}^{-1}$ de $\mathrm{N}$, divididos em três aplicações conforme recomendações da CQFS-RS/SC (2004), durante os meses de novembro, dezembro e janeiro para os tratamentos T1 e T2. Já nos tratamentos T3 e T4, a adubação nitrogenada foi parcelada em seis aplicações quinzenais de novembro a janeiro. Na safra 2013-2014 foram aplicados $100 \mathrm{~kg} \mathrm{ha}^{-1}$ de $\mathrm{N}$ e $150 \mathrm{~kg}$ ha $^{-1}$ de $\mathrm{K}_{2} \mathrm{O}$, sendo que nos tratamentos com adubação convencional (T1 e T2) a adubação foi parcelada em três aplicações nos meses de dezembro, janeiro e fevereiro. A fertirrigação (T3 e T4), foi parcelada em seis aplicações quinzenais, de novembro a janeiro. Para a aplicação da fertirrigação os tratamentos receberam água durante uma hora e meia, sendo meia hora para o enchimento completo das mangueiras com água, meia hora para a aplicação da solução nutritiva e ao final mais meia hora para limpeza das mangueiras e dos gotejadores, o que equivale a $4 \mathrm{~mm}$ de lâmina de água.

Em 2012, foram coletadas amostras de solo da área total, pois ainda não havia sido implantado o sistema de irrigação, e todas as plantas receberam o mesmo tratamento. Já em 2013, foram coletadas amostras de solo a uma profundidade de 0 a $20 \mathrm{~cm}$, em quatro pontos distintos de cada parcela experimental. As análises químicas de solo foram realizadas de acordo com a metodologia descrita por TEDESCO et al. (1995) para determinar o $\mathrm{pH}$ e os teores de $\mathrm{P}, \mathrm{K} \mathrm{Ca}$ e Mg.

Ao final da estação de crescimento da safra 2012-2013, foi medida a altura das plantas. Nos anos de 2012 e 2013, durante o período hibernal, também foi medida a circunferência do tronco a $15 \mathrm{~cm}$ acima do ponto de enxertia. Quantificou-se o número de esporões, brindilas curtas (comprimento menor que $15 \mathrm{~cm}$ ) e brindilas longas (comprimento maior que 15 $\mathrm{cm})$ no inverno de 2013.

A coleta de folhas foi realizada na segunda quinzena de janeiro, no ano de 2014, sendo coletadas no terço médio de ramos do ano, nos quatro quadrantes da planta, totalizando 40 folhas por amostra. As análises do tecido foliar foram realizadas pelo método da digestão seca, para a extração dos elementos químicos $\mathrm{Ca}, \mathrm{Mg}, \mathrm{P}, \mathrm{K}, \mathrm{Fe}, \mathrm{Zn}$ e $\mathrm{Mn}$, conforme descrito por EMBRAPA (2009). O B foi determinado pelo método Azometina- $\mathrm{H}$, e o $\mathrm{N}$, por arraste de vapores utilizando o aparelho semimicro-Kjeldahl, de acordo com TEDESCO et al. (1995).

Por se tratar de plantas jovens, os frutos foram avaliados apenas na safra 2013-2014. No início de novembro de 2013 realizou-se o raleio de frutos em todas as parcelas, sendo estimada uma produtividade em torno de $12 \mathrm{t} \mathrm{ha}^{-1}$. Logo antes da colheita, realizouse a contagem e a coleta de 15 frutos por parcela para análise. No início de abril de 2014 os frutos de cada parcela foram colhidos e pesados. O rendimento médio por hectare foi calculado multiplicando o rendimento médio por planta pelo número de plantas por hectare.

Para compor a amostra da análise química de polpa foram retiradas duas fatias longitudinais em lados opostos de 15 frutos, as quais foram trituradas ainda frescas para a posterior digestão. Para determinar $\mathrm{Ca}, \mathrm{Mg}$ e $\mathrm{K}$ utilizou-se a metodologia da digestão seca. Após a digestão seca, adicionou-se $15 \mathrm{ml}$ de $\mathrm{HCl}$ $1,8 \mathrm{~N}$ (extrato original). Neste extrato determinouse os teores de $\mathrm{Ca}$ e $\mathrm{Mg}$ por espectrofotometria de absorção atômica, e os teores de $\mathrm{K}$ por fotometria de chama. Para extrair $\mathrm{N}$ e $\mathrm{P}$ utilizou-se digestão úmida, com $\mathrm{H}_{2} \mathrm{SO}_{4}$ e $\mathrm{H}_{2} \mathrm{O}_{2}$. Após a digestão determinou-se $\mathrm{P}$ pelo método de MURPHY\& RILEY (1962), e o N por arraste de vapor, utilizando aparelho semimicroKjeldahl.

Nos frutos foram avaliados o teor de sólidos solúveis ( ${ }^{\circ}$ Brix) e o teor de sólidos solúveis com refratômetro digital. A maturação foi determinada pelo índice iodo-amido (1-5), sendo que 1 representa que o fruto está imaturo e apresenta elevado teor de amido (fruto completamente corado), e 5 quando o fruto está maduro e o amido está totalmente hidrolisado (fruto não corado). Para quantificar a firmeza de polpa utilizou-se penetrômetro manual, o qual mediu a resistência da polpa à inserção do êmbolo de $11 \mathrm{~mm}$. A acidez titulável (AT) foi determinada pela titulação de 5,0 mL de suco com $\mathrm{NaOH} 1 \mathrm{~mol} \mathrm{~L}^{-1}$ até o ponto de viragem $(\mathrm{pH} \mathrm{8,1)} \mathrm{e} \mathrm{os} \mathrm{resultados} \mathrm{foram} \mathrm{expressos}$ em \% de ácido málico.

A incidência de 'bitter pit' foi avaliada pela presença ou ausência de lesões necróticas na epiderme 
dos frutos amostrados.

Os dados foram submetidos à análise de variância $(\mathrm{p} \leq 0,05)$ através do teste de Duncan, com o programa SAS 6.08 (SAS, 1996).

\section{RESULTADOS E DISCUSSÃO}

Nas duas safras acompanhadas durante o experimento, foram identificados diversos períodos de déficit hídrico, sendo que em alguns momentos a tensão no solo chegou a valores extremamente baixos, como em dezembro de 2013 quando atingiu $-65 \mathrm{kPa}$. Nos tratamentos irrigados manteve-se a tensão no solo em -10kPa durante o período de avaliações.

Na safra 2012-2013, quando da implantação do experimento, não houve diferença entre os tratamentos quanto ao teor dos macronutrientes $\mathrm{Ca}, \mathrm{Mg}, \mathrm{K}$ e $\mathrm{P}$ no solo, e também em relação ao $\mathrm{pH}$. Portanto, a forma física de aplicação dos fertilizantes (convencional e via fertirrigação), e a adição ou não de água via irrigação, não influenciaram a fertilidade do solo. Todos os valores estavam dentro da faixa normal ou acima dela (CQFS-RS/SC 2004).

Os aspectos vegetativos avaliados nos anos de 2012 e 2013, circunferência de tronco, altura de plantas e número de estruturas produtivas (esporão, brindila curta e longa), não foram influenciados pela forma de aplicação do fertilizante e pelo uso ou não de irrigação (dados não apresentados).
Na safra 2013-2014 os teores foliares de $\mathrm{Mg}$ nos tratamentos com adubação convencional foram superiores aos dos tratamentos fertirrigados (Tabela 1). Observou-se diferença significativa quanto à forma de aplicação do fertilizante para $\mathrm{K}$ e $\mathrm{Fe}$, havendo incremento nos teores nos tratamentos fertirrigados. Verificou-se deficiência de B em todos os tratamentos $\left(<25 \mathrm{mg} \mathrm{kg}^{-1}\right)$ sendo que a testemunha (T1) foi o tratamento mais deficiente para este nutriente. $\mathrm{O}$ uso de irrigação e/ou fertirrigação proporcionou teores significativamente superiores para o B no tecido vegetal. Para os demais nutrientes, não houve diferença entre os tratamentos quanto ao uso de irrigação.

Quanto à composição mineral dos frutos, os teores de $\mathrm{N}, \mathrm{P}$ e $\mathrm{Mg}$ não diferiram significativamente entre as formas de aplicação dos fertilizantes, nem tampouco à adição ou não de água via irrigação (Tabela 2). Os maiores níveis de $\mathrm{K}$ foram observados nos tratamentos submetidos a fertirrigação, sendo que apenas a adição de água via irrigação não influenciou o teor deste nutriente na polpa dos frutos. NEILSEN et al. (2004) observaram que a fertirrigação contendo $\mathrm{K}$ (15 g por planta) aumentou a concentração de K nas folhas e nos frutos, e consequentemente, a acidez dos frutos em quatro cultivares de maçãs avaliadas. Potássio é o nutriente mais acumulado nos frutos, seguido por $\mathrm{N}$ e P (NACHTIGALL \& DECHEN 2006).

Tabela 1 - Teores foliares de macronutrientes e micronutrientes em função da forma física de aplicação dos fertilizantes (convencional e via fertirrigação) e da adição ou não de água via irrigação. Safra 20132014.

Table 1- Leaves levels of macronutrients and micronutrients as a function of the physical form of application of fertilizers (conventional solid soil fertilization and fertigation) and the addition or not of water irrigation. 2013-2014 growing season.

\begin{tabular}{|c|c|c|c|c|c|c|c|c|c|}
\hline \multirow{2}{*}{ Tratamentos } & $\mathrm{N}$ & $\mathrm{P}$ & $\mathrm{K}$ & $\mathrm{Ca}$ & $\mathrm{Mg}$ & $\mathrm{Fe}$ & $\mathrm{Mn}$ & $\mathrm{Zn}$ & B \\
\hline & \multicolumn{5}{|c|}{$\left(\mathrm{g} \mathrm{kg}^{-1}\right)$} & \multicolumn{4}{|c|}{$\left(\mathrm{mg} \mathrm{kg}^{-1}\right)$} \\
\hline $\mathrm{T} 1$ & $24,6 a$ & $2,8 \mathrm{a}$ & $9,5 b$ & $10,4 a$ & $3,2 a$ & $74,1 \mathrm{~b}$ & $402,2 a$ & $78,2 \mathrm{a}$ & $10,5 b$ \\
\hline $\mathrm{T} 2$ & $23,2 \mathrm{a}$ & $2,8 \mathrm{a}$ & $11,2 b$ & $9,5 \mathrm{a}$ & $3,1 \mathrm{a}$ & $77,6 b$ & $424,3 \mathrm{a}$ & $82,0 \mathrm{a}$ & $13,9 \mathrm{a}$ \\
\hline $\mathrm{T} 3$ & $24,9 \mathrm{a}$ & $2,7 \mathrm{a}$ & $18,9 \mathrm{a}$ & $9,0 \mathrm{a}$ & $2,6 b$ & $94,7 \mathrm{a}$ & $419,1 \mathrm{a}$ & $78,6 \mathrm{a}$ & $16,9 a$ \\
\hline $\mathrm{T} 4$ & $23,0 \mathrm{a}$ & $2,8 \mathrm{a}$ & $16,9 \mathrm{a}$ & $9,5 \mathrm{a}$ & $2,7 \mathrm{~b}$ & $86,8 \mathrm{a}$ & $399,7 \mathrm{a}$ & $77,1 \mathrm{a}$ & $14,7 \mathrm{a}$ \\
\hline
\end{tabular}

T1= Adubação convencional; T2= adubação convencional + irrigação; T3= Irrigação + fertirrigação; T4= fertirrigação. Médias seguidas pela mesma letra na coluna não diferem entre si quando comparadas pelo teste de Duncan $(\mathrm{p}<0,05)$.

$T 1=$ Conventional fertilization; $T 2=$ conventional fertilization + irrigation; $T 3=$ fertigation

+ irrigation; $T=4$ fertigation. Average followed by the same letter in the column do not differ when compared by Duncan test $(p<0,05)$. 
Tabela 2 - Teores de macronutrientes na polpa de maçãs, e comparação das médias por contrastes em função da forma física de aplicação dos fertilizantes (convencional e via fertirrigação) e da adição ou não de água via irrigação. Safra 2013-2014.

Table 2 - Levels of macronutrients in apple fruit flesh, in function of the physical form of application of fertilizers (conventional solid soil fertilization and fertirrigation) and the addition or not of water irrigation. 2013-2014 growing season.

\begin{tabular}{cccccc}
\hline \multirow{2}{*}{ Tratamentos } & $\mathrm{N}$ & $\mathrm{P}$ & $\mathrm{K}$ & $\mathrm{Ca}$ & $\mathrm{Mg}$ \\
\cline { 2 - 5 } & \multicolumn{5}{c}{$\left(\mathrm{mg} \mathrm{kg}^{-1}\right)$} \\
\cline { 3 - 5 } & $689 \mathrm{a}$ & $186 \mathrm{a}$ & $668 \mathrm{~b}$ & $81,5 \mathrm{a}$ & $74,4 \mathrm{a}$ \\
$\mathrm{T} 1$ & $662 \mathrm{a}$ & $132 \mathrm{a}$ & $652 \mathrm{~b}$ & $73,9 \mathrm{ab}$ & $78,3 \mathrm{a}$ \\
$\mathrm{T} 2$ & $607 \mathrm{a}$ & $130 \mathrm{a}$ & $930 \mathrm{a}$ & $62,8 \mathrm{~b}$ & $80,3 \mathrm{a}$ \\
$\mathrm{T} 3$ & $654 \mathrm{a}$ & $134 \mathrm{a}$ & $904 \mathrm{a}$ & $71,4 \mathrm{ab}$ & $80,7 \mathrm{a}$
\end{tabular}

$\overline{\mathrm{T} 1=}$ Adubação convencional; $\mathrm{T} 2=$ adubação convencional + irrigação; T3= Irrigação+ fertirrigação; T4= fertirrigação. Médias seguidas pela mesma letra na coluna não diferem entre si quando comparadas pelo teste de Duncan $(\mathrm{p}<0,05)$.

$T 1=$ Conventional fertilization; $T 2=$ conventional fertilization + irrigation; $T 3=$ fertigation + irrigation; $T 4$ fertigation. Average followed by the same letter in the column do not differ when compared by Duncan test $(p<0,05)$.

Para o Ca nos frutos, a testemunha (adubação convencional sem irrigação) apresentou o maior teor deste nutriente em relação ao tratamento irrigação + fertirrigação, não diferindo dos demais tratamentos. Isto se deve ao menor tamanho dos frutos, maior teor de matéria seca (KILILI et al. 1996), e consequentemente à maior concentração de solutos. Considerando os valores críticos de macronutrientes na polpa que podem comprometer a qualidade póscolheita em maçãs $\left(\mathrm{Ca}\right.$, inferior a $40 \mathrm{mg} \mathrm{kg}^{-1}$; $\mathrm{P}$, inferior a $100 \mathrm{mg} \mathrm{kg}^{-1} ; \mathrm{K}$, superior a $950 \mathrm{mg} \mathrm{kg}^{-1} ; \mathrm{Mg}^{\text {, }}$ superior a $40 \mathrm{mg} \mathrm{kg}^{-1}$ e $\mathrm{N}$ superior a $500 \mathrm{mg} \mathrm{kg}^{-1}$ ) (TERBLANCHE 1981, apud AMARANTE et al. 2012), observou-se que apenas Mg e N estão acima dos valores críticos para a preservação da qualidade póscolheita, sugerindo que estes frutos podem apresentar distúrbios fisiológicos durante a armazenagem.

Os tratamentos fertirrigados apresentaram maior relação $\mathrm{K} / \mathrm{Ca}$ e $(\mathrm{K}+\mathrm{Mg}) / \mathrm{Ca}$ na polpa quando comparados aos tratamentos com adubação convencional, em consequência dos altos teores de $\mathrm{K}$ nos tratamentos fertirrigados (dados não apresentados). Não houve influência da irrigação nestes parâmetros avaliados $(\mathrm{K} / \mathrm{Ca}$ e $(\mathrm{K}+\mathrm{Mg}) / \mathrm{Ca})$. Segundo NACHTIGALL \& FREIRE (1998), relações $\mathrm{K} / \mathrm{Ca},(\mathrm{K}+\mathrm{Mg}) / \mathrm{Ca}$ altas resultam em frutos com maior susceptibilidade ao "bitter pit". A relação $\mathrm{N} / \mathrm{Ca}$ não diferiu significativamente entre os tratamentos. Este atributo é mais importante para determinar o grau de incidência e severidade de "bitter pit", do que o teor isolado de $\mathrm{Ca}$ nos frutos, sendo recomendado valor abaixo de 15 (AMARANTE et al. 2010). Neste estudo a relação $\mathrm{N} / \mathrm{Ca}$ foi inferior a $15 \mathrm{em}$ todos os tratamentos.

$\mathrm{Na}$ avaliação dos frutos na colheita, o teor de sólidos solúveis e índice iodo-amido não apresentaram diferença significativa entre os tratamentos, independente da forma de aplicação dos fertilizantes e o uso ou não de irrigação (Tabela 3).

A adubação convencional proporcionou frutos com maior firmeza de polpa provavelmente devido ao menor calibre dos frutos (Tabela 3 ). A concentração de materiais de parede celular e a firmeza da polpa podem diminuir com o aumento do tamanho dos frutos, devido ao alongamento celular excessivo (TAIZ \& ZEIGER 2004). Frutos submetidos à irrigação são maiores, não por possuir mais células, e sim células maiores. Células grandes são possivelmente as responsáveis pela redução na firmeza de polpa (ATKINSON et al. 1998), pois os espaços intercelulares são maiores.

Os tratamentos fertirrigados apresentaram maior acidez titulável em comparação à adubação convencional. Isto se deve ao maior teor de $\mathrm{K}$ nos frutos dos tratamentos fertirrigados, pois quanto 
maior a concentração vacuolar de cátions, maior a concentração de ácidos orgânicos que não estão facilmente acessíveis para 'quebra' durante a respiração (HUNSCHE et al. 2003). Frutos com menores teores de $\mathrm{K}$ apresentam menor calibre e acidez titulável, mas em contrapartida têm maior firmeza de polpa (HUNSCHE et al. 2003). KILILI et al. (1996) relataram que a restrição hídrica no final do ciclo favorece a coloração, o teor de sólidos solúveis e a firmeza de polpa. A firmeza de polpa, assim como a acidez titulável, são parâmetros determinantes na conservação dos frutos durante o armazenamento refrigerado por longos períodos.

Para a incidência de "bitter pit", não houve diferença significativa entre os tratamentos. A maioria dos distúrbios fisiológicos que ocorrem em maçãs está de alguma forma relacionada com a deficiência de Ca. Além da composição mineral, fatores de natureza genética e metabólica podem interferir na manifestação dos diferentes distúrbios fisiológicos em pós- colheita (AMARANTE et al. 2012).

$\mathrm{O}$ tratamento irrigação + fertirrigação apresentou o maior peso médio de frutos (197 g) na safra 2013-2014 (Tabela 4). A comparação das médias dos tratamentos revelou que houve diferença significativa entre os tratamentos para o peso médio de frutos, indicando que a adubação via fertirrigação foi mais eficiente, pois disponibiliza prontamente os nutrientes na solução do solo quando comparado com a adubação convencional. Diferenças de rendimento ocasionadas pelo uso de fertirrigação tendem a diminuir à medida que as árvores envelhecem (KIPP 1992; NEILSEN et al. 1999). FALLAHI et al. (2008), NEILSEN et al. (2010) e NACHTIGALL et al. (2014) relatam que o suprimento de água via irrigação aumenta o tamanho dos frutos, e consequentemente a produtividade. KIPP (1992), ao comparar o uso de fertirrigação com apenas irrigação, verificou que nos primeiros anos do pomar as plantas fertirrigadas estavam mais bem nutridas, apresentaram mais flores por ramo, e maior produção do que as somente irrigadas.

Para o parâmetro produtividade não houve diferença significativa entre os tratamentos (Tabela 4). VEVERKA \& PAVLACKA (2012) relatam que o uso de irrigação em anos com baixa precipitação pluviométrica proporcionou aumento de mais de $40 \%$ na produtividade, ao passo que nos anos com chuvas regulares o aumento foi de apenas $20 \%$.

Tabela 3 - Avaliação de frutos na colheita em função da forma física de aplicação dos fertilizantes (convencional e via fertirrigação) e da adição ou não de água via irrigação. Safra 2013-2014.

Table 3 - Assessment of fruit at harvest as a function of the physical form of application of fertilizers (conventional solid soil fertilization and fertirrigation) and the addition or not of water irrigation. 2013-2014 growing season.

\begin{tabular}{cccccc}
\hline Tratamentos & $\begin{array}{c}\text { Firmeza } \\
\text { de Polpa } \\
\left(\text { lb pol-2) }^{-2}\right.\end{array}$ & $\begin{array}{c}\text { Sólidos } \\
\text { Solúveis } \\
(\%)\end{array}$ & $\begin{array}{c}\text { Índice de } \\
\text { iodo-amido } \\
(1-5)\end{array}$ & $\begin{array}{c}\text { Acidez } \\
(\mathrm{meq} / 100 \mathrm{~mL})\end{array}$ & $\begin{array}{c}\text { Incidência } \\
\text { de "bitter pit" } \\
(\%)\end{array}$ \\
\hline T1 & $19,7 \mathrm{a}$ & $13,2 \mathrm{a}$ & $3,9 \mathrm{a}$ & $7,8 \mathrm{~b}$ & $2,8 \mathrm{a}$ \\
T2 & $19,4 \mathrm{ab}$ & $13,0 \mathrm{a}$ & $3,9 \mathrm{a}$ & $7,7 \mathrm{~b}$ & $1,3 \mathrm{a}$ \\
T3 & $18,8 \mathrm{~b}$ & $14,0 \mathrm{a}$ & $3,8 \mathrm{a}$ & $9,1 \mathrm{a}$ & $0,4 \mathrm{a}$ \\
T4 & $19,0 \mathrm{~b}$ & $13,1 \mathrm{a}$ & $3,9 \mathrm{a}$ & $9,1 \mathrm{a}$ & $3,6 \mathrm{a}$ \\
\hline
\end{tabular}

T1= Adubação convencional; T2= adubação convencional + irrigação; T3= Irrigação + fertirrigação; T4= fertirrigação.

Médias seguidas pela mesma letra na coluna não diferem entre si quando comparadas pelo teste de Duncan $(\mathrm{p}<0,05)$.

$T 1=$ Conventional fertilization; $T 2=$ conventional fertilization + irrigation $; 3=$ fertigation + irrigation;

T4= fertigation .

Average followed by the same letter in the column do not differ when compared by Duncan test $(p<0,05)$. 
Tabela 4 - Peso médio de frutos e produtividade de macieiras em função da forma física de aplicação dos fertilizantes (convencional e via fertirrigação) e da adição ou não de água via irrigação. Safra 20132014.

Table 4 - Average fruit weight and productivity of apple trees depending on the physical form of application of fertilizers (conventional solid soil fertilization and fertirrigation) and the addition or not of water irrigation. 2013-2014 growing season.

\begin{tabular}{ccc}
\hline \multirow{2}{*}{ Tratamentos } & Peso Médio & Produtividade \\
\cline { 2 - 3 } & $-(\mathrm{g})$ & $\left(\mathrm{t} \mathrm{ha}^{-1}\right)$ \\
\hline T1 & $163 \mathrm{c}$ & $9,84 \mathrm{a}$ \\
T2 & $169 \mathrm{bc}$ & $9,05 \mathrm{a}$ \\
T3 & $197 \mathrm{a}$ & $11,81 \mathrm{a}$ \\
T4 & $182 \mathrm{ab}$ & $10,98 \mathrm{a}$
\end{tabular}

T1= Adubação convencional; T2= adubação convencional + irrigação; T3= irrigação + fertirrigação; T4= fertirrigação. Médias seguidas pela mesma letra na coluna não diferem entre si quando comparadas pelo teste de Duncan $(\mathrm{p}<0,05)$.

$T 1=$ Conventional fertilization; $T 2=$ conventional fertilization

+ irrigation; T3= fertigation + irrigation; T4= fertigation .

Average followed by the same letter in the column do not differ when compared by Duncan test $(p<0,05)$.

\section{CONCLUSÕES}

O uso de irrigação e ou fertirrigação não influenciou o desenvolvimento inicial de macieiras, suas estruturas produtivas e a altura de plantas.

A adição de água via irrigação juntamente com a adubação por fertirrigação proporcionou o desenvolvimento de frutos com maior calibre.

Quanto à aplicação de fertilizantes via fertirrigação, apesar de ter apresentado bons resultados, quanto aos teores foliares, acidez titulável, peso médio de frutos é necessário ajustar as doses e épocas de aplicação.

Pelo fato do experimento ter sido implantado em um pomar jovem, se faz necessário prosseguir com o experimento para a obtenção de resultados mais conclusivos.

\section{REFERÊNCIAS}

AL-YAHYAI R. 2012. Managing irrigation of fruit trees using plant water status. Agric. Sci. 3: 35-43.

AMARANTE CVT et al. 2010. Identificação pré-colheita do risco de ocorrência de 'bitter pit' em maçãs 'Gala' por meio de infiltração com magnésio e análise dos teores de cálcio e nitrogênio nos frutos. Rev. Bras. Frutic. 32: 27-34. AMARANTE CVT et al. 2012. Composição mineral de maçãs 'Gala' e 'Fuji' produzidas no sul do Brasil. Pesq.
Agropecu. Bras. 47: 550-560.

ATKINSON CJ et al. 1998.Temperature and irrigation effects on the cropping development and quality of 'Cox's Orange Pippin' and 'Queen Cox' apples. Sci. Hort. 75: 5981.

COMISSÃO DE QUÍMICA E FERTILIDADE DO SOLO - CQFS-RS/SC. 2004. Manual de adubação e calagem para os estados do Rio Grande do Sul e de Santa Catarina.10 ed. Porto Alegre, 400p.

CONCEIÇÃO MAF et al. 2009. Balanço hídrico na cultura da macieira no Rio Grande do Sul. In: XVI Congresso Brasileiro de Agrometeorologia. Anais... Belo Horizonte, MG.

CONCEIÇÃO MAF et al. 2011. Demanda hídrica e coeficientes de cultura $(\mathrm{Kc})$ para macieiras em Vacaria, RS. Ciênc. Rural. 41: 459- 462.

EMBRAPA. 2009. Manual de análises químicas de solos, plantas e fertilizantes. Brasília, DF: Embrapa Informação Tecnológica, $627 \mathrm{p}$.

FALLAHI E et al. 2008. Effects of irrigation systems and rootstocks on water use, tree growth, fruit quality and mineral nutrients in apples during the third and fourth year after planting. Acta Hort. 772: 33-39.

FALLAHI E et al. 2010. Efficient irrigation for optimum fruit quality and yield in apples. HortScience. 45: 16161619.

FALLAHI E. 2012. Influence of rootstock and irrigation methods on water use, mineral nutrition, growth, fruit yield, and quality in 'gala' apple. HortTechnology. 22: 731-737.

HUNSCHE M et al. 2003. Efeito da adubação potássica na 
qualidade pós colheita de maçãs 'Fuji'. Pesqui. Agropecu. Bras. 38: 489-496.

KILILI AW et al. 1996. Composition and quality of 'Braeburn' apples under reduced irrigation. Sci. Hort. 67: $1-11$.

KIPP JA. 1992. Thirty years fertilization and irrigation in Dutch apple orchards: a review. Fert. Res. 32: 149-156.

MURPHY J \& RILEY JP. 1962. A modified single solution method for the determination of phosphate in natural waters. Anal. Chim. Acta. 27: 31-36.

NACHTIGALL GR \& DECHEN AR. 2006. Seasonality of nutrients in leaves and fruits of apple trees. Sci. Agríc. 63: 493-501.

NACHTIGALL GR \& FREIRE CJS. 1998. Previsão da incidência de 'bitter pit' em maçãs através dos teores de cálcio em folhas e frutos. Rev. Bras. Frutic. 20: 158-166.

NACHTIGALL GR et al. 2014. Viabilidade da fertirrigação em pomares de macieiras no sul do Brasil. Agropecuária Catarinense. 27: 25-33.

NEILSEN D et al. 1998. Using soil solution monitoring to determine the effects of irrigation management and fertigation on nitrogen availability in high density apple orchards. J. Amer. Soc. Hort. Sci. 123: 706-713.

NEILSEN D \& NEILSEN GH. 2008. Fertigation of deciduous fruit trees: apples and sweet cherry. In: IMUS P; PRICE MR (Eds.) Fertigation: Optimizing the utilization of water and nutrition. International Potash Institute, Horgen, Switz, p. 76-88.

NEILSEN D et al. 2010. Effect of irrigation and crop load management on fruit nutrition and quality for 'Ambrosia/ M.9' apple. Acta Hort. 868: 63-72.

NEILSEN GH et al. 1999. Response of soil and irrigated fruit trees to fertigation or broadcast application of nitrogen, phosphorus and potassium. HortTecnology. 9: 393-401.

NEILSEN GH et al. 2004. Response of apple to fertigation of $\mathrm{N}$ and $\mathrm{K}$ under conditions susceptible to the development of K deficiency. J. Amer. Soc. Hort. Sci. 129: 26-31.

PETRI JL. 2006. Formação de flores, polinização e fertilização. In: A Cultura da Macieira. Epagri. Florianópolis, p. 229-260.

SAS INSTITUTE. 1996. The SAS-system for windows: release 6.08 (Software). Cary, 633p.

SOUZA TR de et al. 2006. Dinâmica de íons e acidificação do solo nos sistemas de fertirrigação e adubação sólida na citricultura. Rev. Bras. Frutic. 28: 501-505.

TAIZ L \& ZEIGER E. 2004. Fisiologia Vegetal. 3. ed. Porto Alegre: Artmed, 710p.

TEDESCO MJ et al. 1995. Análise de solo, plantas e outros materiais. 2. Ed. Porto Alegre-RS. Departamento de Solos UFRGS, $174 \mathrm{p}$.

VEVERKA V \& PAVLACKA R. 2012.The effect of drip irrigation on the yield and quality of apples. Acta Univ. Agr. Silv. Mend. Brun. 15: 247-252. 